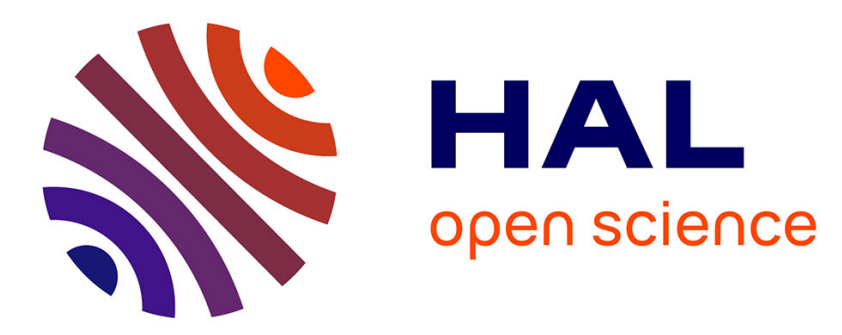

\title{
Model refinements of transformers via a subproblem finite element method
}

Patrick Dular, Patrick Kuo-Peng, Mauricio Valencia Ferreira da Luz, Laurent Krähenbühl

\section{- To cite this version:}

Patrick Dular, Patrick Kuo-Peng, Mauricio Valencia Ferreira da Luz, Laurent Krähenbühl. Model refinements of transformers via a subproblem finite element method. ISEF, Sep 2015, Valencia, Spain. pp.234. hal-01199833

\section{HAL Id: hal-01199833 \\ https://hal.science/hal-01199833}

Submitted on 16 Sep 2015

HAL is a multi-disciplinary open access archive for the deposit and dissemination of scientific research documents, whether they are published or not. The documents may come from teaching and research institutions in France or abroad, or from public or private research centers.
L'archive ouverte pluridisciplinaire HAL, est destinée au dépôt et à la diffusion de documents scientifiques de niveau recherche, publiés ou non, émanant des établissements d'enseignement et de recherche français ou étrangers, des laboratoires publics ou privés. 


\title{
MODEL REFINEMENTS OF TRANSFORMERS VIA A SUBPROBLEM FINITE ELEMENT METHOD
}

\author{
Patrick Dular ${ }^{1}$, Patrick Kuo-Peng ${ }^{2}$, Mauricio V. Ferreira da Luz ${ }^{2}$ and Laurent Krähenbühl ${ }^{3}$ \\ ${ }^{1}$ University of Liege, FNRS, Dept. of Electrical Engineering and Computer Science, ACE, Liege, Belgium \\ ${ }^{2}$ Universidade Federal de Santa Catarina, GRUCAD, Florianopolis, Brazil \\ ${ }^{3}$ Université de Lyon, Ampère (CNRS UMR5005), École Centrale de Lyon, Écully, France
}

\begin{abstract}
A progressive modeling of transformers is performed via a subproblem finite element method. A complete problem is split into subproblems with different adapted overlapping meshes. Model refinements are performed from ideal to real flux tubes, 1-D to 2-D to 3-D models, linear to nonlinear materials, perfect to real materials, single wire to volume conductor windings, and homogenized to fine models of cores and coils, with any coupling of these changes. The proposed unified procedure efficiently feeds each subproblem via interface conditions, which lightens mesh-to-mesh sources transfers, and quantifies the gain given by each refinement on both local fields and global quantities.
\end{abstract}

\section{Introduction}

For efficient and accurate numerical modeling of transformers, an innovative step-by-step methodology is developed. It is based on a finite element (FE) subproblem (SP) method (SPM) with magnetostatic and magnetodynamic problems solved in a sequence on different adapted meshes [1]-[5], from simple 1-D models up to accurate 3-D models, in a large frequency range, of the magnetic circuits and their windings (stranded or massive coils). Each step of the SPM aims at improving the solution obtained at previous steps via any coupling of the following changes, defining model refinements: change from ideal to real (with leakage flux) flux tubes [1], change from 1-D to 2-D to 3-D [2], change of material properties [1]-[3] (e.g., from linear to nonlinear), change from perfect to real materials [4], change from single wire to volume conductor windings [4], [5], and newly developed change from homogenized [6] to fine models (cores as lamination stacks and coils as wire or foil windings, with the details affecting their high frequency behaviors). The methodology involves and couples numerous techniques that have been developed by the authors and, up to now, only applied for simplified test problems [1]-[5]. It can also help in education with a progressive understanding of the various aspects of transformer design.

\section{Progressive Magnetostatic and Magnetodynamic Models}

\section{Methodology}

Transformers are made of magnetic regions, defining magnetic circuits, and active (connected to external electric circuits, i.e., windings or coils) and passive (not fed by circuit, e.g., tank) conducting regions. Such systems are planned to be studied with the following methodology, defining sequences of adequate changes/corrections as these listed in the introduction.

Magnetic circuits - For magnetic cores with possible air gaps, the analysis is first focused on ideal flux tubes neglecting the leakage fluxes and core losses, before considering the coil conductors with their details. A preliminary equivalent magnetic circuit can define a $0-\mathrm{D}$ model. Some of its branches can then be progressively refined via the consideration of their actual geometries, in 1-D, 2-D and 3-D, via SPs of associated dimensions [2]. Such SPs consider changes of boundaries of 
domains that can be either extended or connected together. Each dimension fixes some boundaries through which some assumptions on magnetic flux are considered via some boundary conditions (BCs). A higher dimension modifies such BCs via interface condition (IC) surface sources (SSs) [2]. Changes of material properties can be considered via volume sources (VSs) or SSs when adding, removing, changing or moving some regions [1]-[3]. Nonlinear behaviors of materials can naturally be taken into account as a change from linear to nonlinear material properties.

Stranded and massive conductors - Once the real flux tubes are tackled, the actual geometry of the windings has to be considered, instead of magnetomotive force (MMF) or flux sources. Simultaneously with the IC sources allowing the ideal flux tubes to become permeable [2], the windings are progressively defined at various levels of precision for their geometry and the distribution of the current they carry. They can progress from 1-D to 2-D to 3-D, from single wire to volume FE geometries, and from stranded (uniform current density distributions in their cross sections) to massive conductors, to improve the local field distributions and to accurately render skin and proximity effects [3]-[5]. The global inductive and resistive behaviors are improved as well. Passive conductors (e.g., transformer tank, magnetic shield) can similarly be added and their models improved.

Juxtaposition of thin or wired regions - Lamination stacks in magnetic cores, and wire and foil windings are first classically considered via homogenization models [6] before being locally corrected (in critical regions of interest; in particular for accurate eddy current losses), with an FE SPM derived from [5] and applied in certain thin or wired regions separately with their details (e.g., single conductors with rectangular cross-section and possible oil ducts for cooling; which is usually unfeasible in a direct 3-D approach), coupled to the remaining regions kept homogenized.

Any change that gives a significant effect on the previously solved SPs has to be considered as a source for these. This defines series of corrections on both magnetic circuits and conductor models.

\section{Subproblem Method - Sequence of Subproblems}

A canonical magnetodynamic problem $p$, to be solved at step $p$ of the SPM, is defined in a domain $\Omega_{p}$, with boundary $\partial \Omega_{p}=\Gamma_{p}=\Gamma_{h, p} \cup \Gamma_{b, p}$. The eddy current conducting part of $\Omega_{p}$ is denoted $\Omega_{c, p}$ and the non-conducting one $\Omega_{c, p}{ }^{C}$ (superscript $C$ means "complementary to $\Omega_{c, p}$ in $\Omega_{p}$ "), with $\Omega_{p}=\Omega_{c, p} \cup \Omega_{c, p}{ }^{C}$. Stranded conductors, when homogenized, belong to $\Omega_{\mathrm{s}, p} \subset \Omega_{c, p}{ }^{C}$.

Magnetic field $\boldsymbol{h}_{p}$ and electric current density $\boldsymbol{j}_{p}$ traces are subject to BCs on surfaces $\Gamma_{h, p}$, whereas magnetic flux density $\boldsymbol{b}_{p}$ and electric field $\boldsymbol{e}_{p}$ traces are subject to BCs on surfaces $\Gamma_{b, p}$, i.e.,

$$
\boldsymbol{n} \times\left.\boldsymbol{h}_{p}\right|_{\Gamma_{h, p}}=\boldsymbol{h}_{s, p},\left.\quad \boldsymbol{n} \cdot \boldsymbol{j}_{p}\right|_{\Gamma_{b, p}}=\boldsymbol{j}_{s, p},\left.\quad \boldsymbol{n} \cdot \boldsymbol{b}_{p}\right|_{\Gamma_{b, p}}=\boldsymbol{b}_{s, p}, \quad \boldsymbol{n} \times\left.\boldsymbol{e}_{p}\right|_{\Gamma_{e, p} \subset \Gamma_{b, p}}=\boldsymbol{e}_{s, p}, \quad(1 \mathrm{a}-\mathrm{b}-\mathrm{c}-\mathrm{d})
$$

with $\boldsymbol{n}$ the unit normal exterior to $\Omega_{p}$ and $\boldsymbol{h}_{s, p}, \boldsymbol{j}_{s, p}, \boldsymbol{b}_{s, p}$ and $\boldsymbol{e}_{s, p}$ some given SSs; note that the magnetodynamic problem only defines $\boldsymbol{e}_{p}$ in $\Omega_{c, p}$, with $\Gamma_{e, p} \equiv \partial \Omega_{c, p} \cap \Gamma_{h, p}$. When zero, the SSs define homogeneous BCs, in particular usually applied on symmetry planes. Some BCs can result in ICs, i.e., fixed discontinuities $[\cdot]_{\gamma_{p}}=\left.\left.\cdot\right|_{\gamma_{p}}{ }^{+-} \cdot\right|_{\gamma_{p}}$, when applied on some double layer surfaces $\gamma_{p}{ }^{+}$and $\gamma_{p}{ }^{-}$ that are geometrically defined as a single surface $\gamma_{p} \subset \Gamma_{h, p} \cup \Gamma_{b, p}$, with the thin region in between exterior to $\Omega_{p}$ [3]-[5], i.e.,

$$
\left[\boldsymbol{n} \times \boldsymbol{h}_{p}\right]_{\gamma_{p}}=\left[\boldsymbol{h}_{s, p}\right]_{\gamma_{p}}, \quad\left[\boldsymbol{n} \cdot \boldsymbol{j}_{p}\right]_{\gamma_{p}}=\left[\boldsymbol{j}_{s, p}\right]_{\gamma_{p}}, \quad\left[\boldsymbol{n} \cdot \boldsymbol{b}_{p}\right]_{\gamma_{p}}=\left[\boldsymbol{b}_{s, p}\right]_{\gamma_{p}}, \quad\left[\boldsymbol{n} \times \boldsymbol{e}_{p}\right]_{\gamma_{p}}=\left[\boldsymbol{e}_{s, p}\right]_{\gamma_{p}} .
$$

The fields are related via the material relations

$$
\boldsymbol{h}_{p}=\mu_{p}^{-1} \boldsymbol{b}_{p} \text { in } \Omega_{p}, \quad \boldsymbol{j}_{p}=\sigma_{p} \boldsymbol{e}_{p} \text { in } \Omega_{c, p}, \quad \boldsymbol{j}_{p}=\boldsymbol{j}_{s, p} \text { in } \Omega_{s, p} \subset \Omega_{c, p}{ }^{C},
$$

where $\mu_{p}$ is the magnetic permeability and $\sigma_{p}$ is the electric conductivity, both region-wise defined, and $\boldsymbol{j}_{s, p}$ is a given source current density in $\Omega_{\mathrm{s}, p}$.

With the magnetic vector potential $\boldsymbol{a}_{p}$ and electric scalar potential $v_{p}$ defined via

$$
\boldsymbol{b}_{p}=\operatorname{curl} \boldsymbol{a}_{p}, \boldsymbol{e}_{p}=-\partial_{t} \boldsymbol{a}_{p}-\operatorname{grad} v_{p}=-\partial_{t} \boldsymbol{a}_{p}-\boldsymbol{u}_{p},
$$


the Gauss and Faraday equations are strongly satisfied. The $\boldsymbol{a}_{p}$ weak formulation of the magnetodynamic problem is then obtained from the weak form of the Ampère equation, i.e. [3],

$$
\begin{gathered}
\left(\mu_{p}^{-1} \operatorname{curl} \boldsymbol{a}_{p}, \operatorname{curl} \boldsymbol{a}^{\prime}\right)_{\Omega_{p}}-\left(\boldsymbol{j}_{s, p}, \boldsymbol{a}^{\prime}\right)_{\Omega_{s, p}}+\left(\sigma_{p} \partial_{t} \boldsymbol{a}_{p}, \boldsymbol{a}^{\prime}\right)_{\Omega_{c, p}}+\left(\sigma_{p} \boldsymbol{u}_{p}, \boldsymbol{a}^{\prime}\right)_{\Omega_{c, p}} \\
+<\boldsymbol{n} \times \boldsymbol{h}_{p}, \boldsymbol{a}^{\prime}>_{\Gamma_{h, p} \gamma_{p}}+<\left[\boldsymbol{n} \times \boldsymbol{h}_{p}\right]_{\gamma_{p}}, \boldsymbol{a}^{\prime}>_{\gamma_{p}}=0, \quad \forall \boldsymbol{a}^{\prime} \in F_{p}^{1}\left(\Omega_{p}\right),
\end{gathered}
$$

where $F_{p}{ }^{1}\left(\Omega_{p}\right)$ is a curl-conform function space defined on $\Omega_{p}$, gauged in $\Omega_{c, p}{ }^{C}$, and containing the basis functions for $\boldsymbol{a}_{p}$ and for the test function $\boldsymbol{a}^{\prime}$ (at the discrete level, this space is defined by edge FEs; the gauge can be based on the tree-co-tree technique); $(\cdot, \cdot)_{\Omega}$ and $\langle\cdot, \cdot\rangle_{\Gamma}$ denote a volume integral in $\Omega$ and a surface integral on $\Gamma$, respectively, of the product of their field arguments.

A complete problem is aimed at being solved as a sequence of SPs, involving models for magnetic and conducting regions of progressive accuracy and calculating successive additive corrections. A particular studied domain with an adapted FE mesh is defined for each SP. In the following, region names with no SP index $p$ are regions of the complete problem, to be involved in particular SPs.

\section{$\underline{\text { Sequenced Models }}$}

\section{Magnetic Circuits - Ideal Flux Tubes}

The magnetic (conducting or not) core regions $\Omega_{m} \subset \Omega_{p}$, possibly extended with air gaps $\Omega_{g} \subset \Omega_{m}$, can first be considered as ideal flux tubes (IFTs), i.e., with no leakage flux (Fig. 1). Their boundaries $\Gamma_{m}=\partial \Omega_{m}$ are then considered as perfect magnetic walls (usually made of non-connected portions $\Gamma_{m, i}$ in 2-D, with $i$ the portion index). The actual current source regions $\Omega_{s}$ exterior to $\Omega_{m}$ are thus idealized as perfectly wounded current sheets around $\Omega_{m}$.

For the so-defined SP $p \equiv$ IFT, the studied domain is limited to $\Omega_{p} \equiv \Omega_{m}$, with a BC on $\Gamma_{m} \subset \Gamma_{b, p}$ fixing a zero normal trace of the magnetic flux density $\boldsymbol{b}_{p}$ (its zero trace is also fixed on the possible remaining zero-flux symmetry planes $\Gamma_{b, p} \backslash \Gamma_{m}$, together with $\boldsymbol{n} \times \boldsymbol{e}_{p}=0$ on portions of $\Gamma_{m}$ also in $\left.\partial \boldsymbol{\Omega}_{c, p}\right)$. In terms of $\boldsymbol{a}_{p}$, one has the equivalent essential BCs

$$
\boldsymbol{n} \cdot \boldsymbol{b}_{p}\left|\Gamma_{m}=0 \quad \Leftrightarrow \quad \boldsymbol{n} \times \boldsymbol{a}_{p}\right| \Gamma_{m}=\boldsymbol{n} \times \operatorname{grad} w_{p} \mid \Gamma_{m},
$$

where $w_{p}$ is a multivalued surface scalar potential defined on $\Gamma_{m}$. In 3-D, this scalar potential undergoes a constant jump through each of the cut lines making $\Gamma_{m}$ simply connected [2]. This can be defined via discontinuous components $w_{\text {disc, }, l, p}$ acting only on one side of each cut line $c l$ (at the discrete level, in a surface layer of surface FEs on one side of each cut line, with $w_{\text {disc, } c l, p}$ varying from a constant on cut line $c l$ to zero on the other boundary of the layer), to be added to the continuous component $w_{\text {cont, } p}$ to give $w_{p}$. In 2-D, such constant jumps come down to the definition of a constant $\boldsymbol{a}_{p}$ (a kind of floating potential when unknown) on each non-connected portion $\Gamma_{m, i}$ of $\Gamma_{m}$ (Fig. 1). The constant jumps are directly (strongly) related to the unknown magnetic fluxes flowing in $\Omega_{m}$ and are weakly related to the MMFs $F_{c l}$ along each cut line $c l$ via the weak formulation (5). Indeed, when tested with the non-local jump test functions $\boldsymbol{a}_{\text {surf,cl }}^{\prime}=\operatorname{grad}_{\mathrm{s}} w_{\text {disc, }, c l, p}\left(\operatorname{grad}_{\mathrm{s}}\right.$ is the surface gradient) [2], [3], it gives, if $\Omega_{p} \subset \Omega_{c, p}{ }^{C}$ for simplification,

$$
\left(\mu_{p}^{-1} \operatorname{curl} \boldsymbol{a}_{p}, \operatorname{curl} \boldsymbol{a}_{\text {surf }, c l}^{\prime}\right)_{\Omega_{p}}+<\boldsymbol{n} \times \boldsymbol{h}_{p}, \boldsymbol{a}_{\text {surf }, c l}^{\prime}>_{\Gamma_{m}}=0 \text {, with }<\boldsymbol{n} \times \boldsymbol{h}_{p}, \boldsymbol{a}_{\text {surf, }, l}^{\prime}>_{\Gamma_{m}} \equiv F_{c l} .
$$

If domain $\Omega_{p}$ is not a massive conductor, i.e., $\Omega_{p} \subset \Omega_{c, p}{ }^{C}$, the required gauge condition on $\boldsymbol{a}_{p}$ in $\Omega_{c, p}{ }^{C}$, thus also on $\Gamma_{b, p}$, allows to fix potential $w_{\text {cont }, p}$ to zero on $\Gamma_{m} \subset \Gamma_{b, p}$. However, if $\Omega_{p} \subset \Omega_{c, p}$, potential $w_{\text {cont }, p}$ must be kept unknown on $\Gamma_{m}$, because $\boldsymbol{a}_{p}$ is not gauged there. 
Each $\Gamma_{m, i}$ can be considered as the boundary of a slot in a device. The related MMF gathers all the current sources in the slot, for all coils, e.g., primary and secondary coils in a transformer. A slot can be generalized to represent the exterior region, including coils as well (Fig. 1, right).

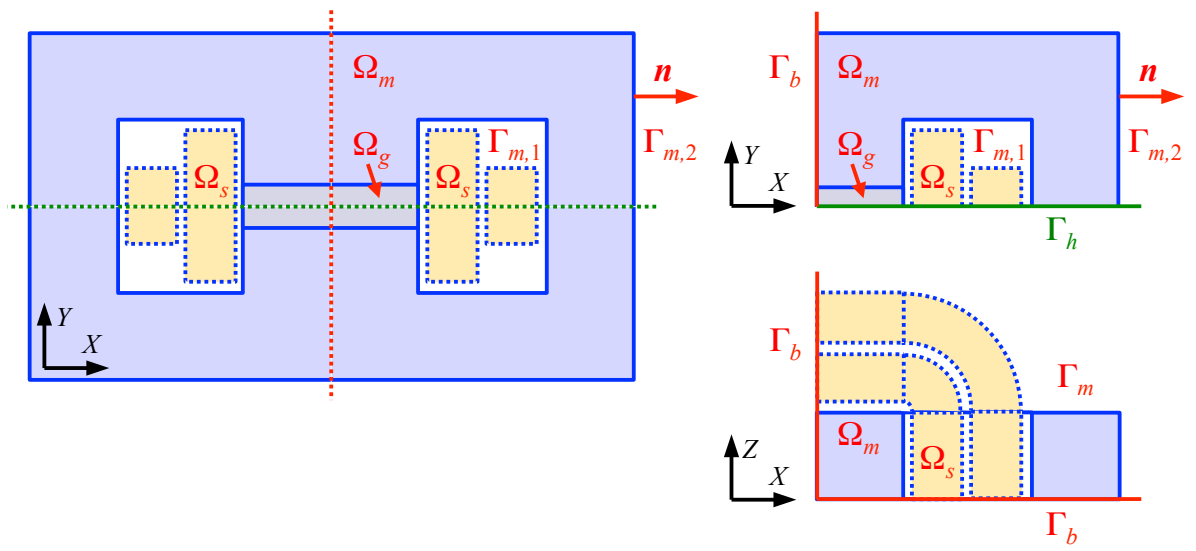

Fig. 1. Shell type transformer: example of a magnetic region $\Omega_{m}$, including a possible air gap $\Omega_{g}$, first considered without leakage flux via perfect magnetic walls BCs on $\Gamma_{m, 1}$ and $\Gamma_{m, 2}$ (left); three symmetry planes allow to reduce the studied domain (right); the end windings need to be considered in 3-D (bottom right).

\section{$\underline{\text { Stranded and Massive Conductors - Real Flux Tubes with Leakage Fluxes }}$}

Volume FE models of the windings are necessary for the accurate determination of their characteristics (e.g., impedances, losses, forces, leakage flux). These can define additional SPs aiming at correcting IFT models, with associated progressive geometrical or physical models.

An IFT solution $q$ is by construction discontinuous through the considered flux walls $\Gamma_{m}$, with a non-zero trace $\boldsymbol{n} \times\left.\boldsymbol{h}_{q}\right|_{\Gamma_{m}}{ }^{+}$on $\Omega_{m}$-side $\Gamma_{m}{ }^{+}$and a zero trace $\boldsymbol{n} \times \boldsymbol{h}_{q} \mid \Gamma_{m}{ }^{-}$on the other side $\Gamma_{m}{ }^{-}$(or nonzero too in case $\Omega_{m}{ }^{C} \subset{ }^{C} \Omega_{p}$, which can be a useful extension) (Fig. 2); trace $\boldsymbol{n} \cdot \boldsymbol{b}_{q}$ is however continuous because zero on both sides. Its correction via an SP $p \equiv$ RFT (real flux tube), that consists in changing the flux wall to a permeable surface allowing leakage flux, can be done by suppressing the discontinuity of $\boldsymbol{n} \times \boldsymbol{h}_{q}$ through the considered flux walls $\Gamma_{m}$ and by simultaneously adding the actual windings (or some simplified models to be further corrected). The opposite of this discontinuity thus serves as an IC-SS [5] for $\left[\boldsymbol{n} \times \boldsymbol{h}_{p}\right]_{\Gamma_{m}}$ weakly defined in (5), whereas $\boldsymbol{n} \cdot \boldsymbol{b}_{p}$ and thus $\boldsymbol{n} \times \boldsymbol{a}_{p}$ remain continuous, i.e.,

$$
\left[\boldsymbol{n} \times \boldsymbol{h}_{p}\right]_{\Gamma_{m}}=-\left[\boldsymbol{n} \times \boldsymbol{h}_{q}\right]_{\Gamma_{m}}=-\boldsymbol{n} \times\left.\boldsymbol{h}_{q}\right|_{\Gamma_{m}}{ }^{+}, \quad\left[\boldsymbol{n} \times \boldsymbol{a}_{p}\right]_{\Gamma_{m}}=0 .
$$

In practice, at the discrete level, the studied domain $\Omega_{p}$ for SP $p \equiv$ RFT includes the windings with their neighborhood, thus also comprising the magnetic cores previously considered via an IFT model (Fig. 2). At this step, if a adequately fine mesh is required for the windings and their vicinity, a coarse mesh can usually be considered for the magnetic core, in which the main flux has already been taken into account with SP $q \equiv$ IFT with a mesh allowing a good accuracy level. Indeed, the leakage flux, flowing in some parts of the magnetic cores, usually slightly modifies the IFT flux. If it is not the case, an additional SP can correct the flux distribution in the cores.
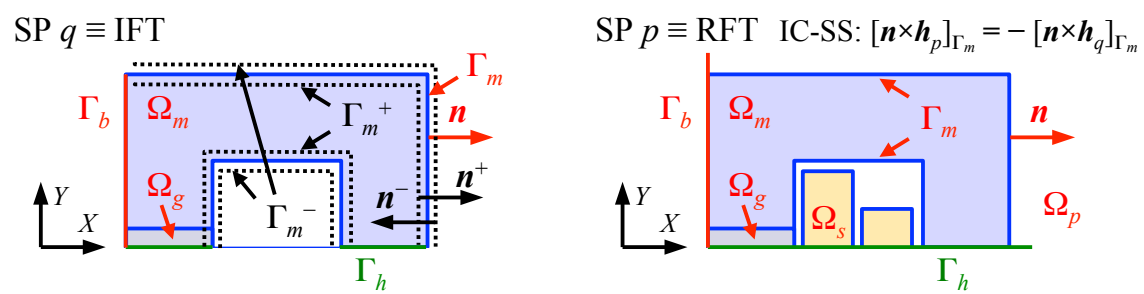

Fig. 2. From IFT model (left) to RFT model (right) with leakage flux via an IC-SS. 
The core could even be considered as a perfect magnetic material (PMM) in a prior SP $r \equiv \mathrm{CORE}$ PMM, defined in $\Omega_{r} \equiv \Omega \backslash \Omega_{m}$ with BC $\boldsymbol{n} \times\left.\boldsymbol{h}_{r}\right|_{\Gamma_{m}}=0$, the solution of which giving a non-zero trace $\boldsymbol{n} \times \boldsymbol{a}_{r} \mid \Gamma_{m}{ }^{-}$. SP $p \equiv$ RFT would then combine the solutions of SPs $q$ and $r$, in complementary domains $\Omega_{q}$ and $\Omega_{r}$, as SSs for ICs, with no need to include the windings already considered in SP $r$, i.e.,

$$
\begin{aligned}
& {\left[\boldsymbol{n} \times \boldsymbol{h}_{p}\right]_{\Gamma_{m}}=-\left[\boldsymbol{n} \times\left(\boldsymbol{h}_{q}+\boldsymbol{h}_{r}\right)\right]_{\Gamma_{m}}=-\left(\boldsymbol{n} \times\left.\boldsymbol{h}_{q}\right|_{\Gamma_{m}}{ }^{+}-\boldsymbol{n} \times\left.\boldsymbol{h}_{r}\right|_{\Gamma_{m}}{ }^{-}\right)=-\boldsymbol{n} \times\left.\boldsymbol{h}_{q}\right|_{\Gamma_{m}}{ }^{+},} \\
& {\left[\boldsymbol{n} \times \boldsymbol{a}_{p}\right]_{\Gamma_{m}}=-\left[\boldsymbol{n} \times\left(\boldsymbol{a}_{q}+\boldsymbol{a}_{r}\right)\right]_{\Gamma_{m}}=-\left(\boldsymbol{n} \times\left.\boldsymbol{a}_{q}\right|_{\Gamma_{m}}{ }^{+}-\boldsymbol{n} \times \boldsymbol{a}_{r} \mid \Gamma_{\Gamma_{m}}{ }^{-}\right) .}
\end{aligned}
$$

A correction from one dimension (SP $q$ ) to the next one (1-D to 2-D or 2-D to 3-D) in an SP $p$ uses again SS-ICs (8a-b) or (9a-b). Indeed, a lower dimension model actually defines some perfect magnetic walls that are made permeable when increasing the dimension [2].

\section{Volume Regions - Changes of Geometrical and/or Physical Properties}

When progressive geometrical models or changes of physical properties are considered for a region, e.g., from a simplified winding geometry in a SP $q$ (via a Biot-Savart or FE model) to the actual FE volume one $\Omega_{v o l, p}$ in an SP $p$, or when changing a portion of a flux tube to an air gap, each change can be done via the following SP $p \equiv$ VOL-REGION-FE.

The general key is to consider such an SP $p$ as the superposition of two SPs, $p$ a and $p$ b, actually simultaneously solved (Fig. 3). SP $p$ a aims at removing the approximate solution $q$ in the newly defined region $\Omega_{v o l, p}$ while keeping it unchanged outside, simultaneously, in FE SP $p$ b, with the consideration of the actual properties $\mu_{p}$ and $\sigma_{p}$ in $\Omega_{v o l, p}$. SP $p$ a exactly cancels out, with no need of any solving, the VSs that would be needed [1]-[3]. It rather leads to trace discontinuities of its direct solution through $\Gamma_{v o l, p}$ given by the corresponding traces of solution $q$ on $\Gamma_{v o l, p}$ as IC-SSs, i.e. (inner minus outer field traces; normal $\boldsymbol{n}$ exterior to $\Omega_{v o l, p}$ ),

$$
\left[\boldsymbol{n} \times \boldsymbol{h}_{p \mathrm{a}}\right]_{\Gamma_{v o l, p}}=-\boldsymbol{n} \times\left.\boldsymbol{h}_{q}\right|_{\Gamma_{v o l, p}} ^{+}, \quad\left[\boldsymbol{n} \times \boldsymbol{a}_{p \mathrm{a}}\right]_{\Gamma_{v o l, p}}==-\boldsymbol{n} \times\left.\boldsymbol{a}_{q}\right|_{\Gamma_{v o l, p}}{ }^{+} .
$$

Because SP $p \mathrm{~b}$ defines a continuous solution through $\Gamma_{v o l, p}$, the trace discontinuities (10a-b) remain valid for the resulting SP $p$, i.e.,

$$
\left[\boldsymbol{n} \times \boldsymbol{h}_{p}\right]_{\Gamma_{v o l, p}}=-\boldsymbol{n} \times\left.\boldsymbol{h}_{q}\right|_{\Gamma_{v o l, p}}{ }^{+}, \quad\left[\boldsymbol{n} \times \boldsymbol{a}_{p}\right]_{\Gamma_{v o l, p}}==-\boldsymbol{n} \times\left.\boldsymbol{a}_{q}\right|_{\Gamma_{v o l, p}}{ }^{+} .
$$
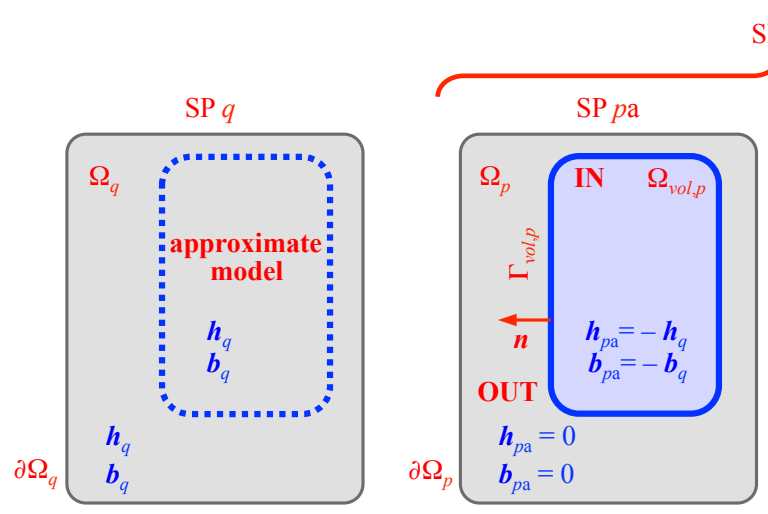

$\overbrace{}^{\operatorname{SP} p}$

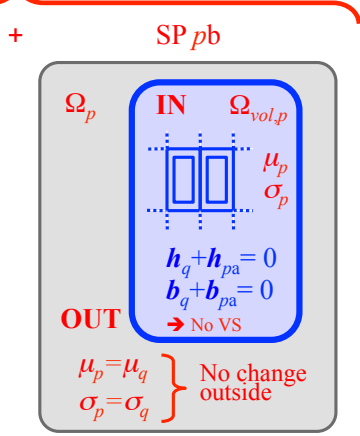

Fig. 3. FE correction (SP $p$ ) of an approximate solution (SP $q$ ): SP $p$ is split into SPs $p$ a and $p$ b, simultaneously solved, SP $p$ a removing the approximate solution $q$ inside the region $\Omega_{v o l, p} \subset \Omega_{p}$ to be geometrically improved and SP $p$ b considering the improved model with its actual geometry and properties.

Note that in all cases with ICs, the studied domain covers both sides of the involved interface, that are therefore naturally coupled. Especially for $\Gamma_{v o l, p}$, this is absolutely different than limiting $\Omega_{p}$ to $\Omega_{v o l, p}$ with a classical BC on $\Gamma_{v o l, p}$, fixing either $\boldsymbol{n} \times \boldsymbol{h}_{p}$ or $\boldsymbol{n} \times \boldsymbol{a}_{p}$ equal to the corresponding trace of the solution of SP $q$. This solution should then be more or less the true solution on $\Gamma_{v o l, p}$, which is in general not true. The corrections, supported by different adapted meshes, are usually of local 
nature, which allows some simplifications of the outer regions, possibly replaced by equivalent circuits extracted from previous SPs.

Each volume correction gives the total field in $\Omega_{v o l, p}$, is discontinuous through $\Gamma_{v o l, p}$ and usually quickly decreases outside, which allows the use of a coarse mesh in the outer region. A volume correction can also be applied in the proposed unified form for a change from a linear to a nonlinear property, and for a newly added region (e.g., tank or shield) in a field solution of an SP $q$, thus acting as a source field in an SP $p$.

\section{Juxtaposition of Thin or Wired Regions - Homogenization to Fine Models}

A homogenization model of core lamination stacks or windings is usually based on assumptions that neglect fringing effects at lamination or winding borders [6]. Some local corrections can be done via SPs for a certain number of laminations or winding turns (Fig. 4), at some particular positions, e.g., where significant edge effects occur. For each correction SP $p \equiv$ VOL-FINE-FE, the studied domain $\Omega_{p}$ comprises the candidate thin or wired regions for correction, now defined with their actual volumes $\Omega_{c, p}$ surrounded by their insulating layers $\Omega_{n c, p}$, defining $\Omega_{v o l, p}=\Omega_{c, p} \cup \Omega_{n c, p}$, that can be finely meshed, and the other thin or wired regions kept homogenized, so coarsely meshed, together with a well chosen neighborhood.

Because SP $p \equiv$ VOL-FINE-FE implies changes of both geometrical and physical properties, it can directly be defined via the method previously developed for SP $p \equiv$ VOL-REGION-FE. Each correction SP is thus again defined via IC-SSs, with the interesting advantage to correct the solution in the actual laminations or windings in $\Omega_{v o l, p}$ while keeping a coupling with the still homogenized region exterior to $\Omega_{v o l, p}$. Here also, using classical BCs on $\Gamma_{v o l, p}$ instead of ICs would generally lead to inaccurate corrections. From the newly calculated total field in $\Omega_{v o l, p}$, a more accurate determination of losses can be obtained.

Corrections of other additional regions can use, as IC-SSs, the updated outer solutions from previous corrections, i.e., the homogenized solution plus the results of these corrections. This is of importance when significant proximity effects occur between laminations or windings.
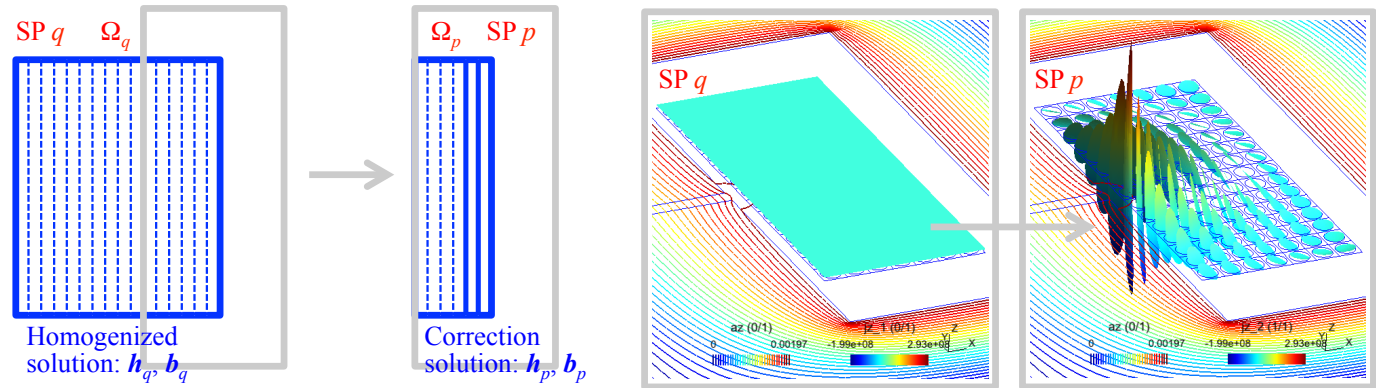

Fig. 4. Local corrections (SP $p$ ) of an homogenized solution (SP $q$ ) for some laminations (e.g., 2 outer laminations) (left) or winding turns (from uniform to non-uniform current density; right).

\section{Weak and Strong SS-ICs - Common Tools}

The sources of the developed correction SPs are all defined via ICs of similar forms (8a-b), (9a-b) or (11a-b). These use parts of SP solutions at previous steps (step $q$, step $r$; a step can gather the updated results of a sequence of previous corrections) as SSs in a new SP $p$. Each chosen pair (a-b) of ICs defines constraints of weak and strong natures.

ICs of type (a) are of weak nature, being involved in a surface integral term in weak formulation (5), defined on a double layer surface $\gamma_{p}$. In all cases, this term becomes

$$
<\left[\boldsymbol{n} \times \boldsymbol{h}_{p}\right]_{\gamma_{p}}, \boldsymbol{a}^{\prime}>_{\gamma_{p}}=<-\boldsymbol{n} \times \boldsymbol{h}_{q}, \boldsymbol{a}^{\prime}>_{\gamma_{p}^{+}}, \text {with } \gamma_{p} \equiv \Gamma_{m} \text { or } \Gamma_{v o l, p}
$$


and is non-zero only for test functions $\boldsymbol{a}^{\prime}$ with a non-zero trace on $\gamma_{p}$. The right hand side of (12) is to be weakly expressed from (5) now applied to SP $q$, i.e., from volume integrals usually limited to

$$
<-\boldsymbol{n} \times \boldsymbol{h}_{q}, \boldsymbol{a}^{\prime}>_{\gamma_{p}^{+}}=\left(\mu_{q}^{-1} \operatorname{curl} \boldsymbol{a}_{q}, \operatorname{curl} \boldsymbol{a}^{\prime}\right)_{\Omega_{p}=\Omega_{q}} .
$$

At the discrete level, the volume integral in (13) is limited to the single layer $E_{T L, p}$ of FEs touching $\gamma_{p}$, on side $\Omega_{m}$ or $\Omega_{v o l, p}$, because it involves only the associated trace $\boldsymbol{n} \times\left.\boldsymbol{a}^{\prime}\right|_{\gamma_{p}}{ }^{+}$having a contribution only in $E_{T L, p}$. The source $\boldsymbol{a}_{q}$, initially in the mesh of SP $q$, has to be projected in the mesh of SP $p$ only in $E_{T L, p}$. This significantly decreases the computational effort of the projection process. An $L_{2^{-}}$ projection is used [1]-[3].

ICs of type (b) are of strong nature, in the sense that they are to be directly defined in function space $F_{p}{ }^{1}\left(\Omega_{p}\right)$ via a fixed discontinuity of the tangential component of $\boldsymbol{a}_{p}$, obtained from the previous solution(s). This can be done via a discontinuous component $\boldsymbol{a}_{\mathrm{disc}, p}$ of $\boldsymbol{a}_{p}$, with support only on one side of the interface, added to the classical continuous component. At the discrete level, this consists in using for $\boldsymbol{a}_{\mathrm{disc}, p}$ additional edge basis functions only on $\gamma_{p}$ that are by definition non-zero only in the previously defined FEs layer $E_{T L, p}$. The result of the projection of $\boldsymbol{a}_{q}$ in $E_{T L, p}$ can also be used to fix $\boldsymbol{a}_{\mathrm{disc}, p}$. The discontinuity can simply be zero, like in (8b).

\section{Application}

The validation and quantification of various SP steps are done on a shell type transformer problem, with a magnetic core $\Omega_{m}(6 \mathrm{~cm} \times 5 \mathrm{~cm} \times 2 \mathrm{~cm}$, leg width $1 \mathrm{~cm}$, relative permeability 500$)$, a possible air gap ( 0 to $3 \mathrm{~mm}$ ), and primary (thickness $4 \mathrm{~mm}$, height $2.7 \mathrm{~cm}$, gap with core center leg $1 \mathrm{~mm}$, 240 turns, fixed voltage at phase 0 at $50 \mathrm{~Hz}$ ) and secondary coils (thickness $3 \mathrm{~mm}$, height $2.7 \mathrm{~cm}$, gap with core primary coil $1 \mathrm{~mm}, 24$ turns, open or short-circuit tests) in $\Omega_{s}$. Symmetry conditions allow to reduce the studied domain for 1-D, 2-D and 3-D analyses (Fig. 5).

The magnetic core with a possible air gap is first considered as an IFT in 1-D, 2-D and 3-D in an open-circuit test, before some correction RFT SPs, applying in various ways some developed SP tools for dimension and model changes; also for material property changes when introducing an air gap; various other correction SPs are possible as theoretically presented and will be further presented. The transformer is also considered in a short-circuit test, in 1-D, 2-D and 3-D. Studied domains and some significant results are illustrated in Fig. 5, with comparisons of the relative corrections on a particular global quantity, the inductance of the primary coil, that are summarized in table 1.

If the field distribution is already accurate for some simplified 1-D and/or 2-D models, e.g., in the open-circuit test, it is not the case in the short-circuit test, in particular with relative corrections from 2-D to 3-D (significant 3-D effects) that can reach hundreds of percents for impedances if no care is given. Also, the accuracy improvement with a higher dimension model is clear (e.g., up to $35 \%$ ) when increasing the air gap.

\section{Conclusion}

A methodology for the progressive FE modeling of transformers has been developed. Models of different accuracy levels (IFT to RFT, 1-D to 3-D, addition of geometrical details, etc.), possibly coupled, are sequenced in the frame of the SPM, with successive additive corrections supported by different adapted meshes. The way the sources act at each correction step, up to the full models with their actual geometries, has been given a particular care and generalized. For all the considered corrections, the sources are always applied via IC-SSs, thus only needed in layers of FEs along boundaries, which strongly lightens the required mesh-to-mesh projections between SPs. Corrections of fields and global quantities can be obtained step by step with a clear view on their significance, to justify their usefulness or not. 


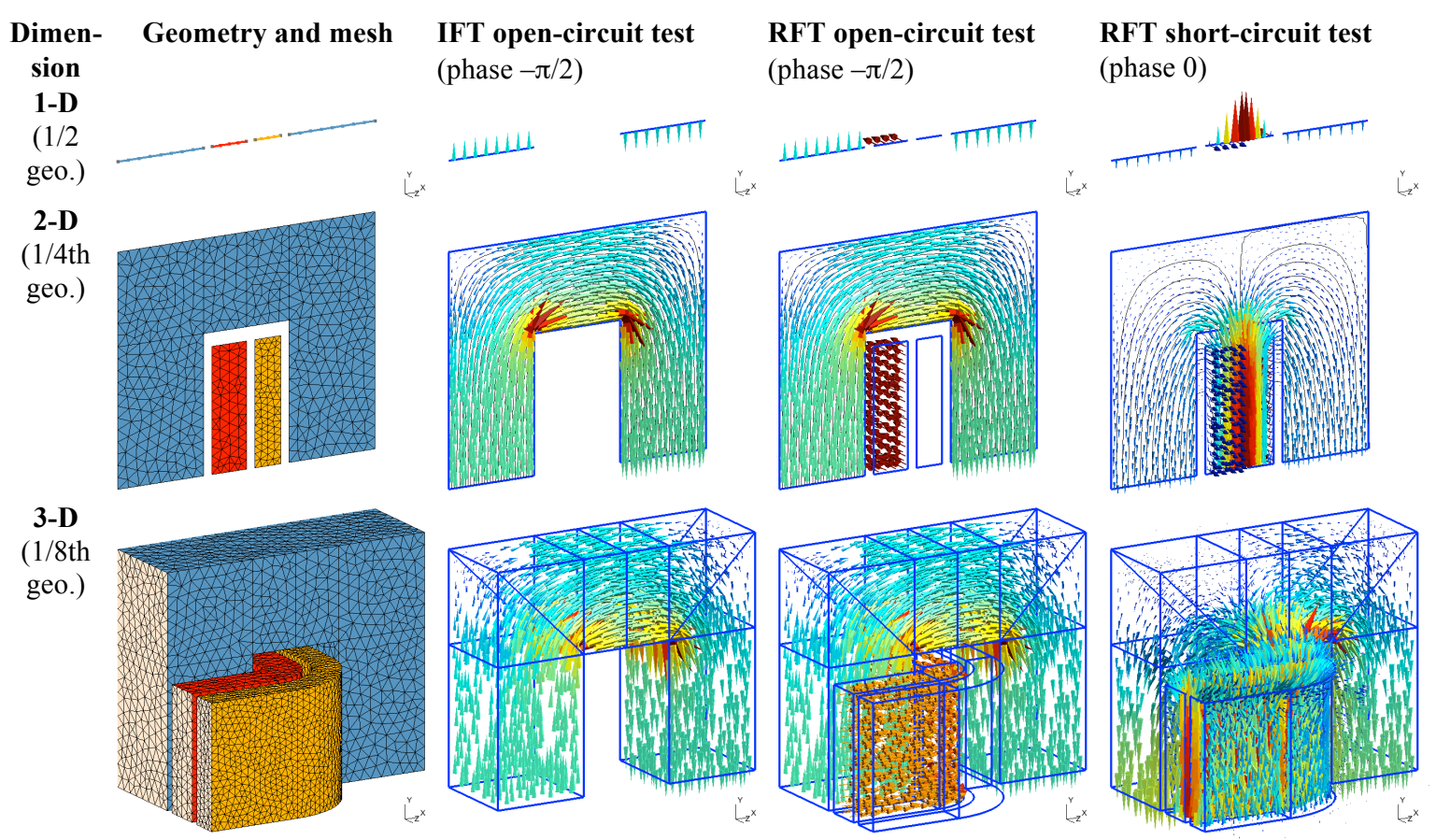

Fig. 5. Shell type transformer with a sequence of 1-D, 2-D and 3-D models with IFT and RFT open and short-circuit tests (arrows: current density, pyramids: magnetic flux density, black lines: field lines (2-D); 2$\mathrm{D}$ and 3-D edge effects are progressively corrected).

TABLE 1. CORRECTIONS OF GLOBAL QUANTITIES: INDUCTANCE OF PRIMARY COIL (CORRECTED VALUES AND RELATIVE CORRECTIONS IN \% WITH RESPECT TO PREVIOUS DIMENSION AND RFT MODEL AFTER IFT MODEL)

\begin{tabular}{|c|c|c|c|c|c|c|}
\hline \multirow{2}{*}{$\begin{array}{c}\text { Air gap } \\
(\mathrm{mm}) \\
0\end{array}$} & \multirow{2}{*}{$\begin{array}{l}\text { Dim. } \\
1-D\end{array}$} & \multicolumn{2}{|c|}{$\begin{array}{l}\text { IFT open-circuit test } \\
\text { Inductance }(\mathrm{mH})\end{array}$} & \multirow{2}{*}{$\begin{array}{l}\text { RFT open-circuit test } \\
\text { Inductance }(\mathrm{mH})\end{array}$} & \multicolumn{2}{|c|}{$\begin{array}{l}\text { RFT short-circuit test } \\
\text { Inductance }(\mathrm{mH})\end{array}$} \\
\hline & & 120.6 & & & 0.1629 & \\
\hline & $2-\mathrm{D}$ & 140.7 & $(+16.6 \%)$ & $141.2(+16.6 \%$, IFT $+0.36 \%)$ & 0.3452 & $(+212 \%)$ \\
\hline & $3-\mathrm{D}$ & 140.7 & $(+0.0 \%)$ & $141.6(+0.23 \%$, IFT $+0.36 \%)$ & 0.9664 & $(+280 \%)$ \\
\hline \multirow[t]{3}{*}{1} & $1-\mathrm{D}$ & 23.39 & & 23.47 & - & \\
\hline & 2-D & 24.05 & $(+2.8 \%)$ & $25.79 \quad(+9.9 \%$, IFT $+7.2 \%)$ & 0.4104 & \\
\hline & $3-\mathrm{D}$ & 24.05 & $(+0.0 \%)$ & $27.58(+6.9 \%$, IFT $+14.7 \%)$ & 1.521 & $(+371 \%)$ \\
\hline \multirow[t]{3}{*}{3} & $1-\mathrm{D}$ & 8.953 & & 8.986 & - & \\
\hline & 2-D & 9.048 & $(+1.1 \%)$ & $10.62(+18.2 \%$, IFT $+17.4 \%)$ & 0.5086 & \\
\hline & $3-\mathrm{D}$ & 9.048 & $(+0.0 \%)$ & $12.26(+15.4 \%$, IFT $+35.5 \%)$ & 2.058 & $(+404 \%)$ \\
\hline
\end{tabular}

\section{References}

[1] P. Dular, R. V. Sabariego, M. V. Ferreira da Luz, P. Kuo-Peng, L. Krähenbühl, "Perturbation finite element method for magnetic model refinement of air gaps and leakage fluxes," IEEE Trans. Magn., vol. 45, no. 3, pp. 1400-1403, 2009.

[2] P. Dular, R. V. Sabariego, C. Geuzaine, M. V. Ferreira da Luz, P. Kuo-Peng and L. Krähenbühl, "Finite element magnetic models via a coupling of subproblems of lower dimensions," IEEE Trans. Magn., vol. 46, no. 8, pp. 2827-2830, 2010.

[3] P. Dular, L. Krähenbühl, R.V. Sabariego, M. V. Ferreira da Luz, P. Kuo-Peng and C. Geuzaine. "A finite element subproblem method for position change conductor systems", IEEE Trans. Magn., vol. 48, no. 2, pp. 403-406, 2012.

[4] P. Dular, V. Péron, R. Perrussel, L. Krähenbühl, C. Geuzaine. "Perfect conductor and impedance boundary condition corrections via a finite element subproblem method," IEEE Trans. Magn., vol. 50, no. 2, paper 7000504, 4 pp., 2014.

[5] P. Dular, V. Péron, L. Krähenbühl, C. Geuzaine. "Subproblem finite element refinement of inductors from wire to static and dynamic volume models," IEEE Trans. Magn., vol. 51, no. 3, paper 7402704, 2015.

[6] L. Krähenbühl, P. Dular, T. Zeidan, F. Buret. "Homogenization of lamination stacks in linear magnetodynamics," IEEE Trans. Magn., vol. 40, no. 2, pp. 912-915, 2004. 